$\begin{array}{cl}\text { PRAMANA } & \text { (C) Indian Academy of Sciences } \\ \begin{array}{c}\text { journal of } \\ \text { physics }\end{array} & \begin{array}{l}\text { Vol. } 70, \text { No. } 5 \\ \text { May } 2008 \\ \text { pp. } 875-886\end{array}\end{array}$

\title{
Scattering of light by a periodic structure in the presence of randomness VII: Application of statistical detection test
}

\author{
V C VANI ${ }^{1, *}$ and S CHATTERJEE ${ }^{2}$ \\ ${ }^{1}$ Department of Instrumentation, Indian Institute of Science, Bangalore 560 012, India \\ ${ }^{2}$ Indian Institute of Astrophysics, 2nd Block, Koramangala, Bangalore 560 034, India \\ ${ }^{*}$ Corresponding author \\ E-mail: vani@isu.iisc.ernet.in; chat@iiap.res.in; chat99@vsnl.com
}

MS received 27 November 2007; accepted 9 January 2008

\begin{abstract}
Detection of periodic structures, hidden in random surfaces has been addressed by us for some time and the 'extended matched filter' method, developed by us, has been shown to be effective in detecting the hidden periodic part from the light scattering data in circumstances where conventional data analysis methods cannot reveal the successive peaks due to scattering by the periodic part of the surface. It has been shown that if $r_{0}$ is the coherence length of light on scattering from the rough part and $\Lambda$ is the wavelength of the periodic part of the surface, the extended matched filter method can detect hidden periodic structures for $\left(r_{0} / \Lambda\right) \geq 0.11$, while conventional methods are limited to much higher values $\left(\left(r_{0} / \Lambda\right) \geq 0.33\right)$. In the method developed till now, the detection of periodic structures involves the detection of the central peak, first peak and second peak in the scattered intensity of light, located at scattering wave vectors $v_{x}=0, Q, 2 Q$, respectively, where $Q=2 \pi / \Lambda$, their distinct identities being obfuscated by the fact that the peaks have width $\Delta v_{x}=2 \pi / r_{0} \gg Q$. The relative magnitudes of these peaks and the consequent problems associated in identifying them is discussed. The KolmogorovSmirnov statistical goodness test is used to justify the identification of the peaks. This test is used to 'reject' or 'not reject' the null hypothesis which states that the successive peaks do exist. This test is repeated for various values of $r_{0} / \Lambda$, which leads to the conclusion
\end{abstract} that there is really a periodic structure hidden behind the random surface.

Keywords. Periodic; random; detection; matched filter; Kolmogorov-Smirnov test.

PACS Nos 42.25.Fx; 42.30.Kq; 42.30.Sy; 02.30.Zz; 02.50.Tt

\section{Introduction}

The detection of a periodic structure, buried in a strongly random surface, by the method of light scattering studies is beset with the problem that the randomness blurs out the distinct peaks in the intensity, expected from diffraction by the periodic part of the surface. If $\Lambda$ is the wavelength of the periodic part and $r_{0}$ is the coherence length of the light on scattering by the random part of the surface, the 


\section{$V C$ Vani and $S$ Chatterjee}

successive peaks are then expected at scattering wave vectors $v_{x}=n Q=n(2 \pi / \Lambda)$ and every peak has a width $\Delta v_{x}=2 \pi / r_{0}$. For high randomness $r_{0}$ is small so that the successive peaks may overlap if $\Delta v_{x} \gg Q$, or $r_{0} \ll \Lambda$ giving rise to overlapping peaks, that are indistinguishable $[1,2]$. The extended matched filtering method, developed by us [3-11] utilizes the well-known conditions that, in the presence of hidden randomness, (1) the peaks ought to have the same shape and (2) the separations between the successive peaks are the same, irrespective of the order of the peaks in the intensity distribution in the scattered data. A similar problem exists in the field of communication engineering, where detection of identically shaped pulses, but arriving at different (unknown, not necessarily periodic) instants of time, and of varying heights is a commonly encountered problem. Our method differs from the conventional matched filtering since in our case, the shapes of the peaks are not known, but are to be selected by the method of least squares from such infinite possibilities. Further, our problem, though seemingly a part of an interdisciplinary field, is greatly compounded by the fact that we are interested in detecting very weak periodic structures in the surface, i.e. $(a / \lambda) \ll 1$, where $a$ is the amplitude of the periodic part. This gives rise to a situation where the central peak subsumes all other peaks and makes them unobservable. For example, in the numerical case that is presented below, the first peak is only $1 / 10$ th and the second peak is $1 / 100$ th of the central one. The matched filter method begins with the hypothesis that the intensity data is actually composed of successive equally spaced and identically shaped peaks and by the method of least squares, determines the best fit for such a hypothesis, as dictated by the physical theory. On the basis of these established theoretical arguments, we have developed a detection method that requires straightforward mathematical deductions and simple computer programming. The results we determine, on comparison with the values of the parameters used in the computation, are found to be very close, thus justifying the strength of the matched filtering method. We had also justified our numerical scheme, by using accurate curve fitting and calculating the reduced $\chi^{2}$ values. This procedure justified all our findings in the detection of the central and the first peak.

The second peak is indeed more elusive, being very weak and thus having the risk of being obfuscated by small errors in calculating the parameters of the system. By very careful numerical work we proved the second peak too to be amenable for detection. The identification and detection of the second peak in the periodic structure proved conclusively the strength of the matched filter method of analysis, when very weak periodic structures are involved. This also brought out the risk of errors in calculating the relative magnitudes of the various peaks and the mathematical and computational limitations associated with the analysis. The fact that the peaks are of widely differing magnitude and that the magnitude of the second peak is more than two orders of magnitude smaller than that of the central peak, makes detection and any quantitative evaluation highly ambiguous and difficult and the problem thus demands very thorough checks for the acceptance of a set of parameters. As explained in the subsequent paragraphs, we have taken the help of well-established statistical significance tests to examine whether the parameters calculated could be relied upon. We have used the Kolmogorov-Smirnov test to show that the probability of the matched filter data coming from the same distribution as the simulated intensity data is quite high. This is indicated if the null 


\section{Scattering of light by a periodic structure}

hypothesis is not rejected, at the 0.05 level of significance. Kolmogorov-Smirnov test has the advantage of being a distribution-free method for testing whether two sample distributions come from the same parent distribution and hence we have used it in the significance analyses in the paper.

In the following section we explain the theory behind the matched filter detection. This has been explained before in our earlier publications, but is explained again for the sake of completeness. The section following this explains in detail about the Kolmogorov-Smirnov statistical test that we have carried out. Following this, we discuss our interpretation. In the final section, we discuss our conclusions and the future course of work.

\section{Theory}

We consider a reflection grating in the $x y$ plane, where elevations in the $z$-direction are given by

$$
\xi(x, y)=a \cos (Q x)+\delta \xi(x, y)
$$

where the random part $\delta \xi(x, y)$ is assumed to be a zero mean Gaussian stationary random process, with

$$
\langle\delta \xi(x, y)\rangle=0, \quad\left\langle\delta \xi(x, y) \delta \xi\left(x^{\prime}, y^{\prime}\right)\right\rangle=\sigma^{2} g(r)
$$

where

$$
r=\left[\left(x-x^{\prime}\right)^{2}+\left(y-y^{\prime}\right)^{2}\right]^{1 / 2} .
$$

We define the wave vector $\mathbf{v}$ of scattering to be

$$
\begin{aligned}
& v_{x}=k\left(\sin \theta_{1}-\sin \theta_{2} \cos \theta_{3}\right), \quad v_{y}=-k \sin \theta_{2} \sin \theta_{3}, \\
& v_{z}=-k\left(\cos \theta_{1}+\cos \theta_{2}\right), \quad v_{x y}^{2}=v_{x}^{2}+v_{y}^{2},
\end{aligned}
$$

where $k=2 \pi / \lambda$ is the wave vector of light, $\lambda$ being the wavelength of light. In what follows, we shall calculate the scattered intensity under the Kirchoff approximation, which is valid for $4 \pi r_{\mathrm{c}} \cos \theta \gg \lambda, r_{\mathrm{c}}$ being the radius of curvature of the surface $[12,13]$. Defining $\left\langle\rho \rho^{*}\right\rangle_{0}$ to be the ratio of the light intensity scattered in the $\left(\theta_{2}, \theta_{3}\right)$ direction to the intensity of light scattered in the specular direction $\left(\theta_{2}=\theta_{1}, \theta_{3}=\right.$ 0 ), for a perfectly smooth surface, we find

$$
\begin{aligned}
\left\langle\rho^{*} \rho\right\rangle_{0}= & \left\{J_{0}^{2}\left(\sqrt{ }\left(2 g_{1}\right)\right) f\left(v_{x}, v_{y} ; g\right)+\sum_{n=1}^{\infty} J_{n}^{2}\left(\sqrt{ }\left(2 g_{1}\right)\right)\left[f\left(v_{x}+n Q, v_{y} ; g\right)\right.\right. \\
& \left.\left.+f\left(v_{x}-n Q, v_{y} ; g\right)\right]\right\} B\left(\theta_{1}, \theta_{2}\right)
\end{aligned}
$$

where, with $\sqrt{ } g=\sigma v_{z} / \sqrt{ } 2$ and $\sqrt{ } g_{1}=a v_{z} / \sqrt{ } 2$, we have

$$
f\left(v_{x}, v_{y} ; g\right)=(2 \pi / A) \int \exp (-g[1-g(r)]) J_{0}\left(v_{x y} r\right) r \mathrm{~d} r
$$


$A$ being the area of the surface in the $x y$ plane.

$$
\begin{aligned}
& B\left(\theta_{1}, \theta_{2}\right)=\left[F_{3}\left(\theta_{1}, \theta_{2}, \theta_{3}\right)\right]^{2} S\left(\theta_{1}, \theta_{2}\right) \\
& \begin{array}{l}
F_{3}\left(\theta_{1} ; \theta_{2}, \theta_{3}\right)=\left(1+\cos \theta_{1} \cos \theta_{2}-\sin \theta_{1} \sin \theta_{2} \sin \theta_{3}\right) / \\
\quad\left(\cos \theta_{1}\left(\cos \theta_{1}+\cos \theta_{2}\right)\right)
\end{array} \\
& S\left(\theta_{1}, \theta_{2}\right)=S\left(\theta_{1}\right) S\left(\theta_{2}\right)
\end{aligned}
$$

with

$$
\begin{aligned}
& S(\theta)=\exp [(-1 / 4) \tan \theta \operatorname{erfc}(K \cot \theta)] \\
& K^{2}=(a Q)^{2}+4(\sigma / l)^{2}
\end{aligned}
$$

of which $F_{3}\left(\theta_{1} ; \theta_{2}, \theta_{3}\right)$ is a geometrical factor and $S\left(\theta_{1}, \theta_{2}\right)$ describes the 'shadowing effect'.

A careful inspection of the above formulae will explain the principles of the matched filter detection process that we describe below. Equation (3) shows that the intensity profile consists of a series of peaks of the same shape, as described by the functions $f\left(v_{x} \pm n Q\right)$, but shifted along the $v_{x}$ axis, the separations between the nearest peaks being always $Q$, where $f\left(v_{x}\right)$ is the shortened notation for $f\left(v_{x}, 0 ; g\right)$. Furthermore, the amplitudes $J_{n}^{2}$ (the argument $\sqrt{ }\left(2 g_{1}\right)$ being dropped from now on) depend purely on $a$ while the shapes of the functions $f\left(v_{x} \pm n Q\right)$ depend totally on the randomness of the system. This is true only in the limit of the elevations being small, and we restrict the numerical calculations in those limits only.

Considering that $\sqrt{ } g_{1} \ll 1$, the amplitudes of the successive $n$th peaks fall as $\left(\left(\sqrt{ } g_{1}\right)^{n} / n !\right)^{2}$ while the width of the peaks vary as $\Delta v_{x} \approx r_{0}^{-1}$, in every case. The coherence length $r_{0}$ of the light due to scattering by randomness is estimated as $\Delta v_{x} \approx r_{0}^{-1}$, where $\Delta v_{x}$ is the half-width at full-maxima of the central peak $f\left(v_{x}\right)$. The separation of the peaks being $\delta v_{x}=Q$, the central $n=0$ peak can submerge all the higher order peaks for $\Delta v_{x} \gg \delta v_{x}=Q$, and the peaks thus become undetectable. The matched filtering method envisages the shape $f\left(v_{x}\right)$ of the central peak and separates the zeroth order peak out from the total intensity profile. This way, it tries to bring out and identify the $n= \pm 1$ peaks, whose shape must match with that of the $n=0$ peak that has been eliminated out. We begin by assuming the shape of the central peak (which must be the same for all other peaks too), with two unknown parameters, $c^{\prime}$ and $y$, to be

$$
f\left(v_{x}\right)=f_{a}\left(v_{x}\right) \equiv\left[1+\left(c^{\prime} / 2 y\right) v_{x}^{2}\right]^{y}
$$

which should be the leading trend for various models of randomness.

We now define

$$
\begin{aligned}
& Z\left(v_{x}\right)=\left[\left\langle\rho^{*}\left(v_{x}\right) \rho\left(v_{x}\right)\right\rangle_{0} /\left\langle\rho^{*}(0) \rho(0)\right\rangle_{0}\right]-f_{a}\left(v_{x}\right) \\
& \chi\left(v_{x}\right)=Z\left(v_{x}\right) / Z_{\max } .
\end{aligned}
$$




\section{Scattering of light by a periodic structure}

We have, in essence, sought to obtain $Z\left(v_{x}\right)$ and $\chi\left(v_{x}\right)$, as quantities, which are essentially $\left\langle\rho^{*}\left(v_{x}\right) \rho\left(v_{x}\right)\right\rangle_{0}$ sans the anticipated central peak. We define the wave vector $Q^{*}$ as that at which $Z\left(v_{x}\right)$ has a maxima. This peak can be identified as the first peak due to scattering by the hidden periodic structure, if and only if its shape matches with the zeroth order peak $f_{a}\left(v_{x}\right)$ that has been eliminated out, this 'matched filtering' being a strict condition that follows from eq. (3).

Further defining $\chi_{a}\left(v_{x}\right)$ as

$$
\chi_{a}\left(v_{x}\right)=\chi_{a N}\left(v_{x}\right) / \chi_{a D}\left(v_{x}\right)
$$

with

$$
\begin{aligned}
& \chi_{a N}\left(v_{x}\right)=\left[f_{a}\left(v_{x}+Q^{*}\right)+f_{a}\left(v_{x}-Q^{*}\right)-2 f_{a}\left(v_{x}\right) f_{a}\left(Q^{*}\right)\right] \\
& \chi_{a D}\left(v_{x}\right)=\left[f_{a}\left(Q^{*}+Q^{*}\right)+f_{a}\left(Q^{*}-Q^{*}\right)-2 f_{a}\left(Q^{*}\right) f_{a}\left(Q^{*}\right)\right],
\end{aligned}
$$

we note that if $f_{a}\left(v_{x}\right)=f\left(v_{x}\right)$, identically, $\Delta\left(v_{x}\right)=\left[\chi\left(v_{x}\right)-\chi_{a}\left(v_{x}\right)\right]$ must vanish identically, in the limit of $\left(J_{2} / J_{0}\right)^{2}$ being extremely small.

The matched filtering is thus effected by using a least square optimization, by defining an estimator

$$
\Delta\left(c^{\prime}, y\right)^{2}=\int_{0}^{Q^{*}}\left|\chi_{a}\left(v_{x}\right)-\chi\left(v_{x}\right)\right|^{2} \mathrm{~d} v_{x}
$$

which can be calculated by computing $\chi\left(v_{x}\right)=\left\{\left[\left\langle\rho^{*}\left(v_{x}\right) \rho\left(v_{x}\right)\right\rangle_{0} /\left\langle\rho^{*}(0) \rho(0)\right\rangle_{0}\right]-\right.$ $\left.f_{a}\left(v_{x}\right)\right\}$ from the experimental data and calculating $\chi_{a}\left(v_{x}\right)$ from eqs (13)-(15) with the assumed $f_{a}\left(v_{x}\right)$. We select the filter by choosing $c^{\prime}$ and $y$ to be the one which gives minimum value for $\Delta^{2}\left(c^{\prime}, y\right)$.

The search for the optimum value of $c^{\prime}$ begins by identifying that $\left(\partial^{2} / \partial v_{x}^{2}\right)\langle\rho \rho\rangle_{0} \approx$ $c^{\prime}$. We next eliminate the central and first-order peaks, and fit the 'residual' to the matched filter parameters. We simulate the scattered data by calculating $\left\langle\rho \rho^{*}\right\rangle_{0}$, from eqs $(3)-(9)$. We next search for the matched filter by choosing a suitable $f_{a}\left(v_{x}\right)$ and calculating $\Delta^{2}$ in every case and thus arriving at the matched filter value $\left(c^{\prime}, y\right)$, where the value of $\Delta\left(c^{\prime}, y\right)^{2}$ is a minimum. The position in the peak in $Z\left(v_{x}\right)$ occurs at $v_{x}=Q^{*}$ from which the value of $Q$ is further approximated as $Q_{\text {cal }}$ and the value of $a$ as $a_{\text {cal }}$, by using the relations (17) and (18), given below.

$$
\begin{aligned}
& f_{a}^{\prime}\left(Q^{*}+Q\right)+f_{a}^{\prime}\left(Q^{*}-Q\right)-2 f_{a}(Q) f_{a}^{\prime}(Q) \\
& \quad \approx\left(J_{2} / J_{1}\right)^{2}\left[f^{\prime}\left(Q^{*}+2 Q\right)+f^{\prime}\left(Q^{*}-2 Q\right)\right] \\
& \quad Z_{\max } J_{0}^{2}-\left[f_{a}(0)+f_{a}\left(2 Q^{*}\right)-2 f_{a}^{2}\left(Q^{*}\right)+2 f_{a}\left(Q^{*}\right) Z_{\max }\right] J_{1}^{2} \\
& \quad \approx\left[f_{a}\left(3 Q^{*}\right)+f_{a}\left(Q^{*}\right)-2 f_{a}\left(2 Q^{*}\right) f_{a}\left(Q^{*}\right)-2 f_{a}\left(Q^{*}\right) Z_{\max }\right] J_{2}^{2} .
\end{aligned}
$$

We calculate $\langle\rho \rho\rangle_{0,1}$ as the sum (3), with only the $n=0,1$ terms, where for $f\left(v_{x}\right)$ we substitute $f_{a}\left(v_{x}\right)$, as obtained by using the matched filter values, while for $a$ we use, $a_{\text {cal }}$ and for $Q$, we use $Q_{\text {cal }}$, being arrived at by using the procedures described in the above paragraph. We then determine 


$$
\delta\left\langle\rho^{*} \rho\right\rangle_{0,1}=\left\langle\rho^{*} \rho\right\rangle_{0}-\left\langle\rho^{*} \rho\right\rangle_{0,1}
$$

The quantity $\delta\left\langle\rho^{*} \rho\right\rangle_{0,1}$ is thus a quantity, in which the $n=0$ and $n=1$ peaks have been eliminated. The next highest term being thus $J_{2}^{2} f\left(v_{x}-2 Q\right)$, this peak must show up at $v_{x}=2 Q$, if it is present. By fine tuning the parameters, $Q_{\text {cal }}, a_{\text {cal }}$, we arrive at the point, where $\int\left|\delta\left\langle\rho^{*} \rho\right\rangle_{0,1}\right|^{2} \mathrm{~d} v_{x}$ is a minimum, where we have chosen the integral to extend from the point of maxima of $\delta\left\langle\rho^{*} \rho\right\rangle_{0,1}$ to the point where it drops to $85 \%$ of this peak value.

Detection of different peaks is beset with the difficulty that the magnitude of the second peak relative to the zeroth peak $\left(J_{2} / J_{0}\right)^{2} \sim 2.1 \times 10^{-3}$ while the magnitude of the first-order peak with respect to the zeroth one $\left(J_{1} / J_{0}\right)^{2} \sim 0.09$. The height of the second peak is thus about one order of magnitude smaller than the first peak and two orders smaller than the zeroth one. Moreover, we have estimated the matched filter with its curvature around its peak at $v_{x}=0$ and tried to verify whether these match with those obtained at $v_{x}= \pm Q^{*}$ and $\pm 2 Q^{*}$. In view of the smallness of $J_{2}^{2}$ with respect to $J_{1}^{2}$ and $J_{0}^{2}$, it is thus necessary to obtain estimates of the function $f\left(v_{x}\right)$ in the tail region also, since the tail of $f\left(v_{x}\right)$ continues well beyond $2 Q^{*}$, as it is found that $f\left(2 Q^{*}\right) / f(0) \gg\left(J_{2} / J_{0}\right)^{2}$. The central theme in all this work is to anticipate the shape of the central peak and hence all other peaks and eliminate the stronger peaks to bring out the identically shaped weaker ones.

\section{The Kolmogorov-Smirnov test}

The Kolmogorov-Smirnov test is a statistical test used to determine whether two different sets of data come from the same parent distribution [14]. The cumulative distribution function is plotted against the data. This test is based on the fact that the two end points of the cumulative distribution data should be at 0 and 1 . The behaviour in between these limits is decided by the individual data. The maximum distance $D$ between the two distributions is the term that enters the calculation of the probability. We also define a significance level $\alpha$, which actually decides the extent of chance occurrence. The null hypothesis $H_{0}$ says that both distributions come from the same parent distribution. If probability $p$ is greater than $\alpha, H_{0}$ is not rejected. Otherwise, it is rejected.

The expressions for calculating the value of probability are given below. If

$$
Q_{k s}(\lambda)=2 \sum_{j=1}^{\infty}(-)^{(j-1)} \exp \left(-2 j^{2} \lambda^{2}\right)
$$

$Q(0)=1, Q(\infty)=0$.

$$
\text { Probability }(D>\text { observed })=Q_{k s}\left(\left[\sqrt{ } N_{e}+0.12+0.11 / \sqrt{ } N_{e}\right] D\right),
$$

where $N_{e}=N_{1} N_{2} /\left(N_{1}+N_{2}\right)$ and $N_{1}$ and $N_{2}$ are the number of points in data sets 1 and 2 respectively. 


\section{Scattering of light by a periodic structure}

Table 1. Basic statistical parameters and parameters of the KolmogorovSmirnov test. $H_{12}$ refers to the null hypothesis when data 1 and 2 are compared. $H_{13}$ refers to the null hypothesis when data 1 and 3 are compared. $A=328 \times 10^{-8}$.

\begin{tabular}{|c|c|c|c|c|c|c|c|c|}
\hline$r_{0} / \Lambda$ & Mean & Median & $\begin{array}{l}\text { Range } \\
\text { difference }\end{array}$ & Std. Dev. & $P$ & KS-d & $\begin{array}{c}H_{12} \\
\alpha=0.05\end{array}$ & $\begin{array}{c}H_{13} \\
\alpha=0.05\end{array}$ \\
\hline 0.10 & 0.978 & 0.977 & $\begin{array}{l}0.0394 \\
0.0013\end{array}$ & 0.009 & 0.07 & 0.18 & 0 & 1 \\
\hline 0.11 & 0.979 & 0.985 & $\begin{array}{l}0.0427 \\
0.0060\end{array}$ & 0.016 & 0.07 & 0.18 & 0 & 1 \\
\hline 0.12 & 0.984 & 0.981 & $\begin{array}{l}0.0673 \\
0.0031\end{array}$ & 0.016 & 0.19 & 0.15 & 0 & 1 \\
\hline 0.13 & 0.986 & 0.986 & $\begin{array}{l}0.0381 \\
0.0003\end{array}$ & 0.012 & 0.05 & 0.19 & 1 & 1 \\
\hline 0.14 & 0.980 & 0.982 & $\begin{array}{l}0.0448 \\
0.0016\end{array}$ & 0.015 & 0.26 & 0.14 & 0 & 1 \\
\hline 0.16 & 0.982 & 0.982 & $\begin{array}{c}0.0438 \\
0\end{array}$ & 0.015 & 0.55 & 0.11 & 0 & 1 \\
\hline 0.18 & 1.000 & 0.996 & $\begin{array}{l}0.0919 \\
0.0037\end{array}$ & 0.023 & 0.44 & 0.12 & 0 & 1 \\
\hline 0.20 & 0.986 & 0.992 & $\begin{array}{l}0.0685 \\
0.0061\end{array}$ & 0.020 & 0.26 & 0.14 & 0 & 1 \\
\hline 0.22 & 0.994 & 0.996 & $\begin{array}{l}0.0961 \\
0.0019\end{array}$ & 0.0235 & 0.19 & 0.15 & 0 & 1 \\
\hline 0.25 & 0.999 & 0.998 & $\begin{array}{l}0.1071 \\
0.0009\end{array}$ & 0.026 & 0.14 & 0.16 & 0 & 1 \\
\hline 0.28 & 0.980 & 0.981 & $\begin{array}{l}0.1146 \\
0.0007\end{array}$ & 0.031 & 0.68 & 0.10 & 0 & 1 \\
\hline 0.32 & 0.990 & 0.997 & $\begin{array}{l}0.1285 \\
0.0069\end{array}$ & 0.035 & 0.34 & 0.13 & 0 & 1 \\
\hline
\end{tabular}

\section{Results}

The results of this paper are summarized in table 1. Studies were carried out at a fixed wavelength of $\lambda=6328 \AA$ and at differing $\left(r_{0} / \Lambda\right)$ varying from 0.10 to 0.32 . The value of $a$ is fixed at $328 \AA$, in the computations.

The simulated intensity data obtained from eq. (3) is taken as the reference data. This is first fitted to the matched filter expression consisting of the zeroorder, first-order and second-order peaks. We then fit the simulated intensity data to a single peak, which means that there is no periodic structure hidden in the random background. The reasoning behind this exercise is the following. Given the intensity data, which appears only as a single broad peak, we have the following two possibilities:

1. There exists a structure within the single peak that the light scattering data has generated (simulated intensity data, in our case).

2. There is no special structure within the intensity data. In this case, the matched filter expression and the structure of periodicity that it produces should be invalid. 

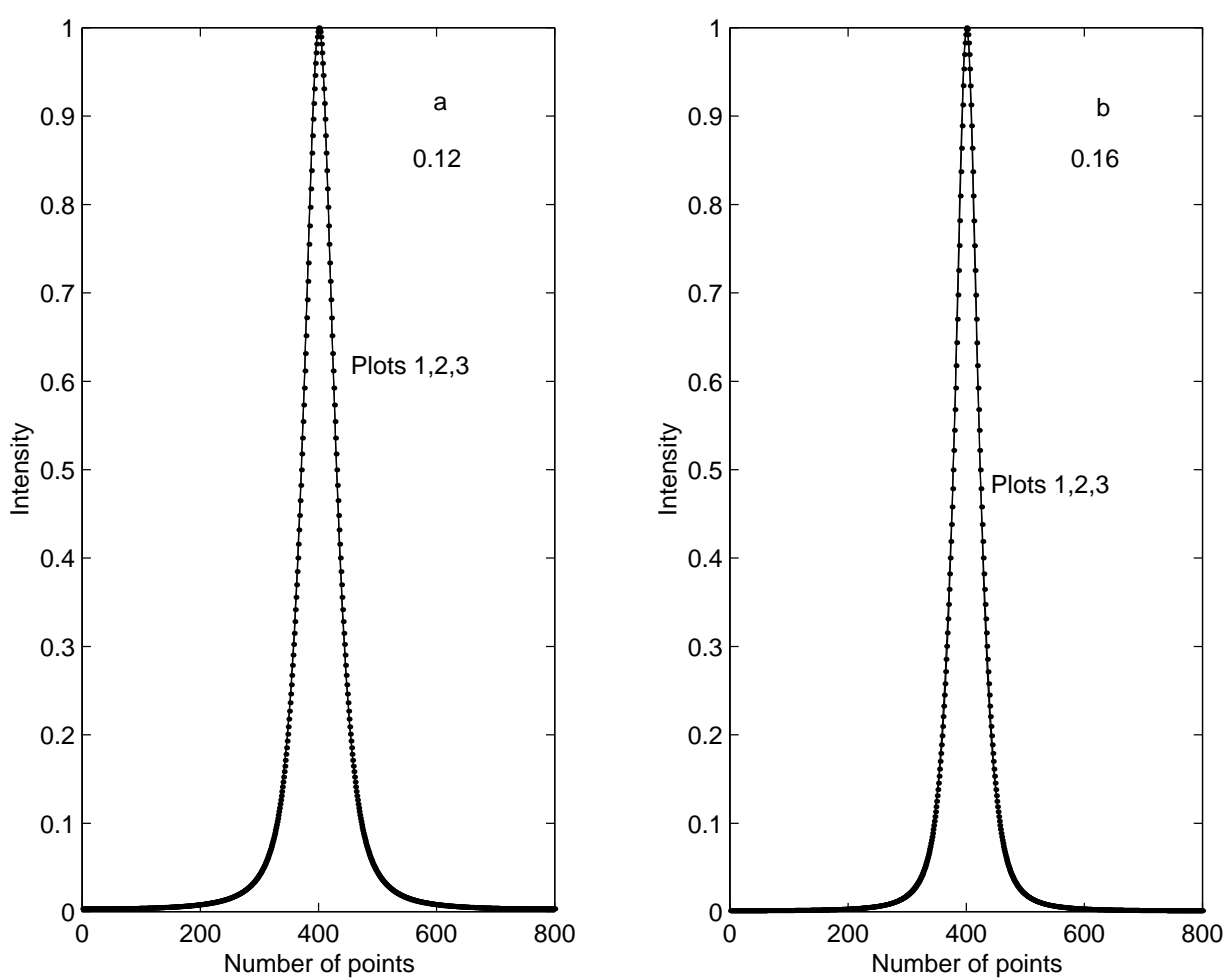

Figure 1. Plot of (1) simulated intensity, (2) intensity as given by the matched filter expression and (3) intensity assuming a single peak. For (a) $r_{0} / \Lambda=0.12$ and $(\mathbf{b}) r_{0} / \Lambda=0.16$.

It is necessary to discriminate between the two cases and statistical methods are employed to test the goodness of fit of the two hypotheses. Firstly, we fit the intensity data to the hypotheses 1 and 2 given above. It is found that the KolmogorovSmirnov test accords equal probability, to be exact, the same probability to both the hypotheses. It is thus necessary not to fit the intensity data as such but to modify the approach, to allow discrimination between the two hypotheses.

We then calculate the following ratios, to help us in deciding the correctness of our theory.

(a) Ratio of the data of option 1 to the reference data [ratio (a)].

(b) Ratio of the data of option 2 to the reference data [ratio (b)].

Our contention is that, if any one of the fits mentioned in options 1 and 2 were exact, the corresponding ratios (a) or (b) should be unity throughout. Unfortunately, this is not the case, and both (a) and (b) deviate from unity at several points. The idea is to determine, as to which of the ratios is closer to unity, throughout the range under study. The reasons for this deviation of the ratio from unity identically, could be the following. The data, as calculated from the theoretical simulation, contains several peaks of decreasing magnitude as the order of the peak increases. Their magnitudes being extremely 'small' they hardly contribute to the 

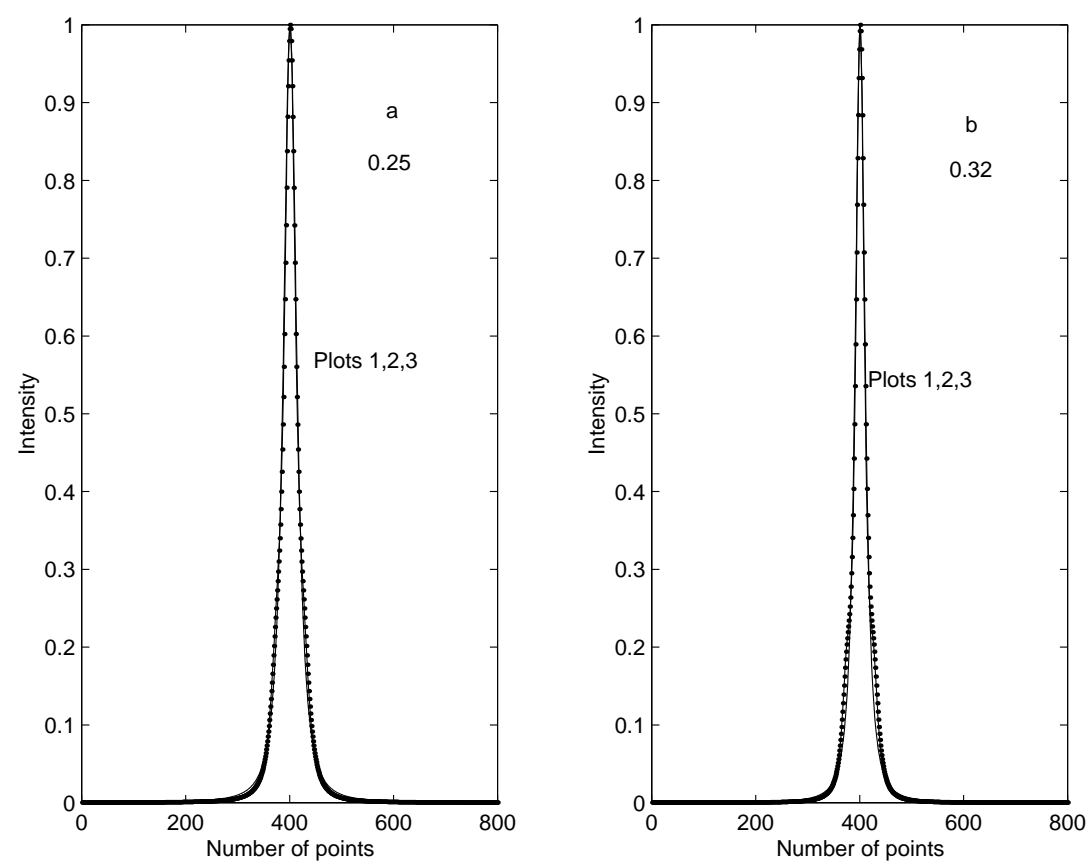

Figure 2. Plot of (1) simulated intensity, (2) intensity as given by the matched filter expression and (3) intensity assuming a single peak. (a) $r_{0} / \Lambda=0.25$ and $(\mathbf{b}) r_{0} / \Lambda=0.32$.

discrimination process unless their contributions and those of the bigger peaks are put on the same footing. To achieve this, we note that though the magnitudes of the higher peaks are very small, they may be comparable to the residuals at the tails. To understand our line of attack, we refer to figures 1 and 2 , which show some of the intensity plots for different values of $r_{0} / \Lambda$. From these plots it is clear that both the matched filter intensity and the single peak intensity fit the reference data quite well. No conclusion can be drawn from these fits as to which one of them resembles the reference data better. Figures 3 and 4 show some of the ratio graphs. From the figures showing the ratio plots, it is quite easy to decide that in the case where the parameter $a$ is not equal to zero, the matched filter intensity resembles the reference data better than the single peak intensity. This conclusion, although impossible from the usual intensity plot, is very clear from a plot of the ratio. This is because, by taking the ratio, we are magnifying the difference at the tails.

To quantify the results, we employ the Kolmogorov-Smirnov statistical goodness test. This test is used to determine whether two sets of data come from the same parent distribution. Here, we use this test to determine whether the data ratio (a) or (b) is from the same distribution as a set of 1's (with a $5 \%$ error allowed, i.e. we do not discard the hypothesis if the ratio lies between 0.95 and 1.05). We are thus following a problem, related to 'estimation of parameters'. The parameter involved here is the ratio and having estimated its value from the data, we proceed to check its deviation from unity. The statistical test involves the following procedure. 

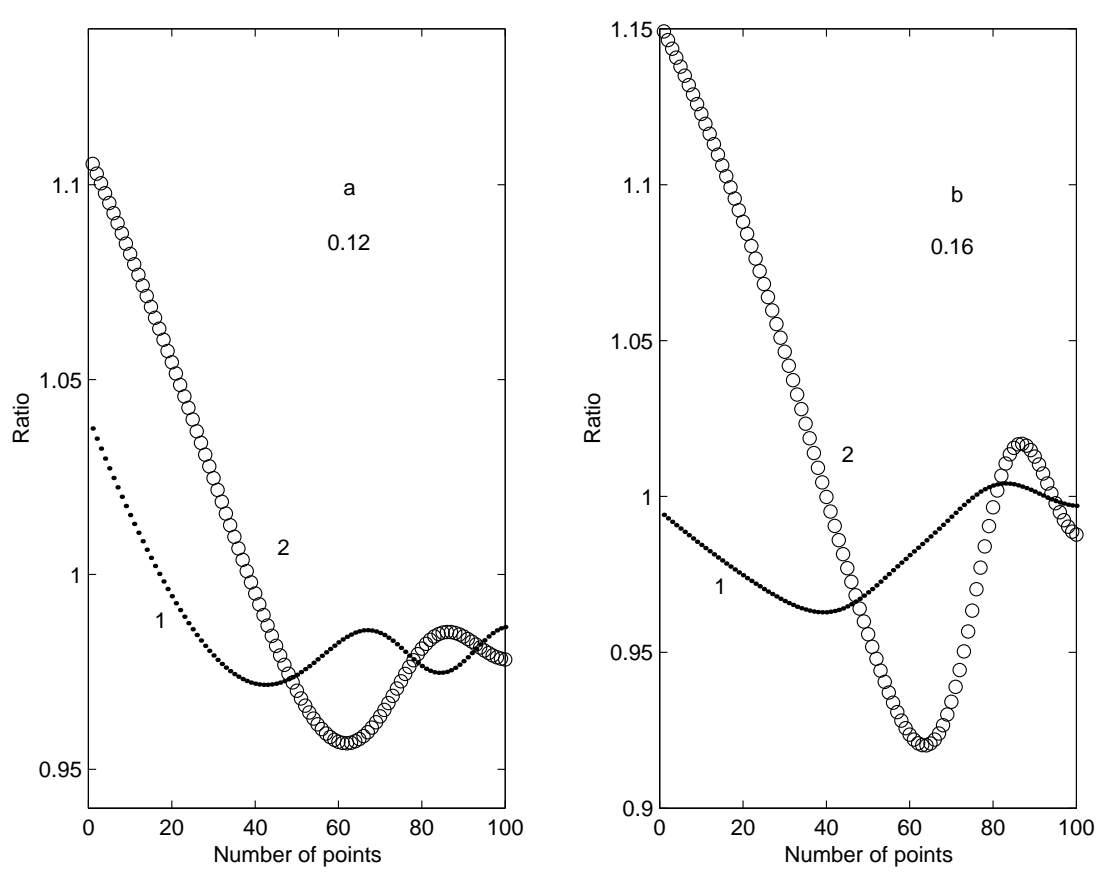

Figure 3. Plot of ratio. (1) Intensity as given by the matched filter expression/simulated intensity (curve 1 with symbol •), (2) intensity assuming a single peak/simulated intensity (curve 2 with symbol o). (a) $r_{0} / \Lambda=0.12$ and (b) $r_{0} / \Lambda=0.16$.

- Arrange data in ascending order.

- Get the cumulative distribution function.

- Define null hypothesis $H_{0}$. In this case the null hypothesis states that the two data are from the same distribution.

- Define significance level. In this case the significance level $\alpha=0.05$.

- We define three sets of data for the Kolmogorov-Smirnov test.

Data 1 - set of units with $5 \%$ error margin

Data 2 - Data ratio (a)

Data 3 - Data ratio (b).

The Kolmogorov-Smirnov test is applied for data 1 and 2 as one case and also data 1 and 3 in the second case.

The results obtained are given in table 1. For the case involving data 1 and 2 the parameters obtained are given in table 1 . Here, the null hypothesis $H_{0}$ is not rejected at the 0.05 significance level. This conclusion is valid for almost all the values of $r_{0} / \Lambda$. In the other case, where data 1 and 3 are involved, the corresponding probabilities are very small, tending to zero in most cases, and we find that the null hypothesis is rejected for almost all the values of $r_{0} / \Lambda$. This is at a significance level of 0.05 . All these observations lead us to conclude that when $a$ is 
Scattering of light by a periodic structure
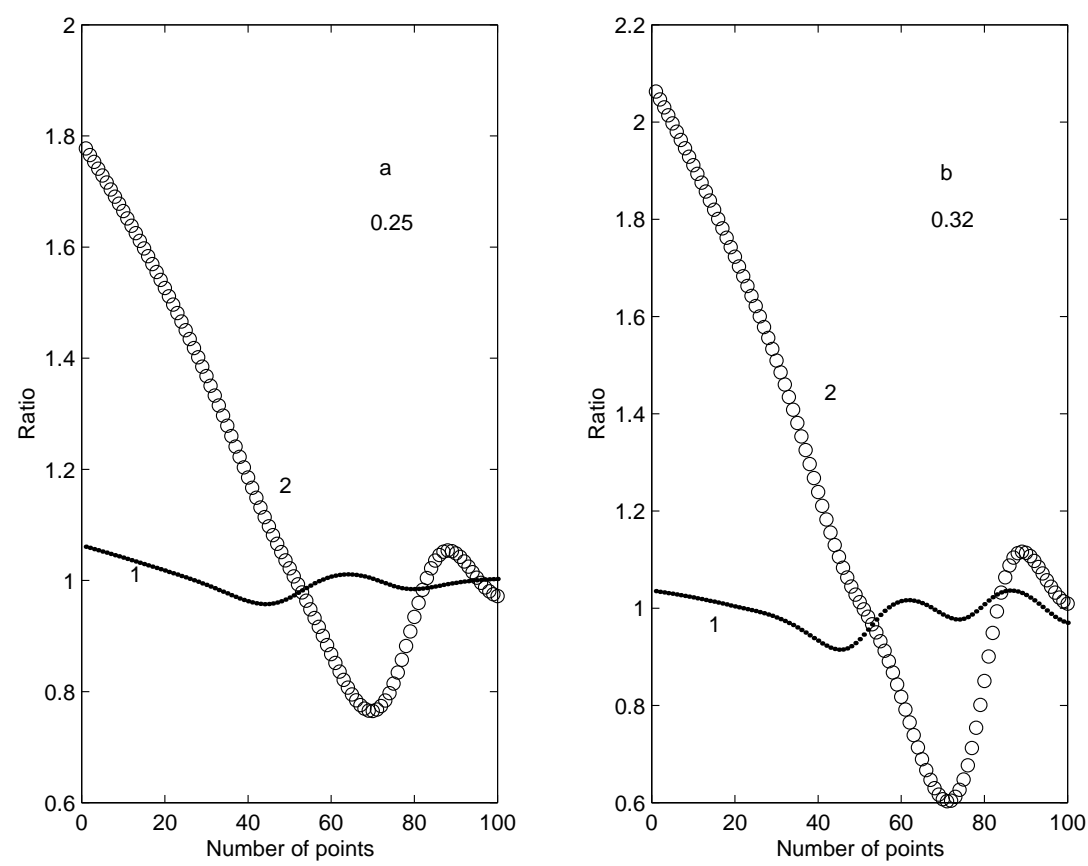

Figure 4. Plot of ratio. (1) Intensity as given by the matched filter expression/simulated intensity (curve 1 with symbol $\bullet$ ), (2) intensity assuming a single peak/simulated intensity (curve 2 with symbol o). (a) $r_{0} / \Lambda=0.25$ and (b) $r_{0} / \Lambda=0.32$.

finite $(a / \lambda \sim 1 / 20)$, there is certainly a periodic structure behind the randomness. This is obvious from the residual plots themselves, but the Kolmogorov-Smirnov test provides a statistical basis for the result.

As the orders of magnitude of the various peaks vary so widely and further the magnitude of the third and fourth peaks should be so small, looking for higher-order peaks becomes impossible. Also, the detection of different peaks as a function of different parameters involved in the matched filter method of detection is still to be carried out. Our subsequent studies will focus on these topics.

\section{Conclusion}

From the results obtained and the subsequent discussion above, we conclude the following. In a situation where the parameter $a$ or the amplitude of the periodic structure is finite, (1) the parameters detected by the matched filter data give a good description of the intensity data, while (2) an expression, consisting of a single broad peak, without any periodic structure, cannot describe the intensity data adequately. This fact is very clearly indicated by the results we have obtained. The null hypothesis is not rejected (for various values of $r_{0} / \Lambda$ ) in the case where a matched filter intensity is chosen and was always rejected when a single peak 
intensity was chosen. This statistical corroboration along with many of our observations explained above, lead us to the above conclusion and further advances the strength of the matched filtering method used by us.

\section{References}

[1] J C Dainty and D Newman, Opt. Lett. 8, 608 (1983)

[2] H P Baltes, H A Ferwerda, A S Glass and B Steinle, Opt. Acta 28, 11 (1981)

[3] S Chatterjee, Indian J. Phys. B74, 363 (2000)

[4] S Chatterjee and V C Vani, Bull. Astron. Soc. India 30, 835 (2002)

[5] S Chatterjee and V C Vani, J. Mod. Opt. 50, 833 (2003)

[6] V C Vani and S Chatterjee, Bull. Astron. Soc. India 31, 457 (2003)

[7] V C Vani and S Chatterjee, Curr. Sci. 86, 177 (2004)

[8] V C Vani and S Chatterjee, Appl. Opt. 46, 3664 (2004)

[9] S Chatterjee and V C Vani, Pramana - J. Phys. 65, 413 (2005)

[10] S Chatterjee and V C Vani, Third International Conference on Inverse Problems: Modeling and Simulation, May 29-June 02, 2006

[11] V C Vani and S Chatterjee, Appl. Opt. 45, 8939 (2006)

[12] P Beckmann and A Spizzichino, The scattering of electromagnetic waves from random surfaces (Pergamon Press, London, 1963)

[13] P Beckmann, Scattering of light by rough surfaces, in Progress in optics edited by E Wolf (North Holland, London, 1967) vol. 6, pp. 55-69

[14] William H Press, Saul A Teukolsky, William T Vellerling and Brain P Flannery, Numerical recipes in Fortran (Cambridge University Press, 1998), published in India by Foundation Book, New Delhi, 1998 